\title{
Geometry, Pattern, Kinetics: Beginning Studio Pedagogy from 2D to 3D
}

\author{
M. Saleh Uddin ${ }^{1}$
}

${ }^{1}$ Department of Architecture, Kennesaw State University, Georgia, USA

\begin{abstract}
Architecture has the strongest educational ties to geometric organization because of the necessity for order and efficiency in construction, three-dimensional space composition, and the desire to create aesthetically pleasing structures. Kimberly Elam, the author of "Geometry of Design" mentions, "Too often as a design professional and educator I have seen excellent conceptual ideas suffer during the process of realization, in large part because of the designer did not understand the visual principles of geometric composition. These principles include an understanding of classic proportioning systems such as the golden section and root rectangles, as well as ratios and proportion, interrelationships of form, and regulating lines." With the primary objective of linking art and architecture through observation, analysis, and composition, this paper demonstrates beginning studio projects where geometry, pattern and kinetics are exercised to enhance understanding of two-dimensional pattern and three-dimensional construction. The objective is to enhance three-dimensional design thinking that has become a challenge in recent trends of questionable dependency on computer modeling applications for design decisions.
\end{abstract}

\section{Introduction}

Visual thinking and the understanding of geometric organization in both $2 \mathrm{D}$ and $3 \mathrm{D}$ are essential in architectural education. This is truer now more than ever before as students tend to depend on digital applications more and lean towards creating surfaces. With the primary objective of linking art and architecture through observation, analysis, and composition, this paper demonstrates beginning studio projects where geometry, pattern and kinetics are exercised to enhance understanding of two-dimensional pattern and three-dimensional construction. This paper promotes the concept of a beginning studio that starts with basic spatial composition of 2D and 3D in design thinking, linking with 3D physical construction.

The assigned projects had four specific goals:

- Observation of random geometry and pattern in nature and man-made objects

- Extraction and modification of geometry to formalize a pattern or a module for pattern

- Design thinking in three dimensions and kinetics, and

- Use of digital software and hardware to fabricate modified pattern in to threedimensional kinetic element for architectural use. 


\section{Pedagogical Context}

In architectural education, the foundation of understanding properties of geometry, composition, scale and proportion in 2D and 3D had been the essential ingredients for design process. They are also complementary to the notion of linking art with architecture. With recent dependency on digital tools in architectural studios there has been a noticeable change in overall understanding of design thinking, three-dimensional exploration, design iteration, and notion of scale and proportion. The confusion of scale in virtual environments and physical prints in this regard is notable.

In this context, it is relevant to note that the formal architectural education was established at the turn of the seventeenth century by the "Beaux-Arts" in France. It emerged in response to the value system at that time and continued to be the only model for two hundred years. Towards the end of the nineteenth century, the value system started to change. A new development of formal design education strongly appeared before World War I in Germany. The Bauhaus model of design education emerged in response to the technological development and industrial revolution. The word "Bauhaus" itself means "house of construction" or "school of building". Bauhaus was founded with the idea of creating a "total" work of art in which all arts, including architecture, would eventually be brought together. The Bauhaus style later became one of the most influential currents in modern design and architectural education.

The general world trend in the late twentieth century caused an emphasis towards scientific, mathematical, and quantitative approach to education, and art education is often refuted because it is based on perception. It is qualitative and subjective which makes it difficult to measure and evaluate. Current inception of digital media and personal computers in architectural education inherently seem to negate the role of art in architecture.

With current dynamic changes in the learning system, and evolving digital means, the design studio pedagogy is in the verge of reformation again after a century. From the academic perspective, Bauhaus principles still seem to be relevant because architecture is always seen as a fine art where principles of formal composition are considered being of greatest importance. Based on this belief, the author is convinced that a beginning studio should start with basic spatial composition of 2D and 3D in design thinking, understanding of geometry, graphic representation, and physical construction.

\section{Geometry, Pattern, Module, Kinetics}

Geometry, pattern, and module are interrelated in their definition, physical characteristics, and architectural interpretation. Discovery of geometry in our surrounding environment is an important learning for all visual designers. This learning includes observation, analysis, and synthesis of geometry for adaptation and reformation. This section of paper looks at definition of Geometry, Pattern, Module, and Kinetics for understanding and their cross relationships.

Geometry (from the Ancient Greek: geo- "earth", -metron "measurement") is a branch of mathematics concerned with questions of shape, size, relative position of figures, and the properties of space. In its simplest form, geometry is the mathematical study of shapes and space. Geometry can deal with flat, two-dimensional shapes, such as squares and circles, or three-dimensional shapes with depth, such as cubes and spheres. Studying geometry is important to develop skills such as logic, problem solving and spatial understanding. Since 
digital applications for drawing and models are based on geometry then it is important to study geometry and graphics of descriptive geometry.

Pattern is a regularity in the world, man-made design, or abstract ideas that repeats in a predictable way. Geometry, form, and shape are primary ingredients of a pattern. Although for architecture pattern is considered visual and structural, it can be behavioral for other fields. Several of our senses may directly be able to observe patterns. The visual element of pattern is constructed by repeating or echoing the elements of an artwork or drawing to communicate a sense of balance, harmony, contrast, rhythm or movement. There are two basic types of pattern in visual studies: Natural Pattern and Man-Made Pattern. Both natural and man-made patterns can be regular or irregular, organic or geometric, structural or decorative, positive or negative and repeating or random.

Natural pattern in visual field is often based on the inspiration we get from observing the natural patterns that occur in nature. We can see these in the shape of a leaf and the branches of a tree, the structure of a crystal, the spiral of a shell, the symmetry of a snowflake and the patterns on animals, fish and insects. Man-made patterns are usually a compositional pattern of lines, shapes, and geometry.

The studio projects illustrated in this paper have been chosen because they all use pattern in an inspirational manner both from nature and man-made.

Module is a self-contained component of a system, often interchangeable, which has a well-defined interface to the other components. Modularity in design is an approach that subdivides a system into smaller parts of modules that can be independently created and then used in different systems. Modularity offers benefits such as reduction in customization with more flexibility in design augmentation.

Kinetics is a new word in architecture borrowed from other disciplines. Wikipedia defines it as a study of motion and its causes. The English Oxford Dictionary defines as, "the branch of chemistry or biochemistry concerned with measuring and studying the rates of reactions". Webster Dictionary defines as, "a branch of science that deals with the effects of forces upon the motions of material bodies or with changes in a physical or chemical system". Historically, architecture has been perceived as a stationary creation and thus the study of kinetics was beyond its scope. In last thirty years, architectural education has integrated various digital tools such as fabrication, analysis, simulation, etc. to replace manual graphics and tactile design thinking and thus integration of kinetics and robotics in beginning studios are not rare.

\section{Projects}

Considering the fact that the recent trend is to design spaces through surface-making in computer modeling program this paper advocates three-dimensional design thinking through pattern and physical kinetic construction. The pattern in this instance is to be observed and analyzed as arts from nature and creation of objects by humans. During this process, students document and analyze texture, composition, pattern, and kinetics to formulate design strategies to create a three-dimensional system with and without kinetics.

Recent inclination of pattern and surface making in digital software by students cannot be ignored. If the understanding of scale, perception of depth, and control of desired geometry can be integrated then results would be more guided than accidental. This paper looks at these following projects where composition is documented as $2 \mathrm{D}$ graphic texture first, and then 
explored in to pattern and 3D construction. Photographs, hand drawings, Photoshop filters, Illustrator outlines, AutoCAD drawing, CAD Laser cut, Rhino 3D model, and STL 3D print are the primary means and techniques of exploration. The final transformation is in threedimensional kinetic construction. The assigned project had their respective criteria and objectives.

\subsection{Project: Observation-Analysis-Synthesis}

All assigned projects started with finding geometry, pattern, and texture from nature and man-made. Figure 1 illustrates student work highlighting observation and documentation from nature before expanding in to a system of geometric network or a structural system in three-dimensional construction.
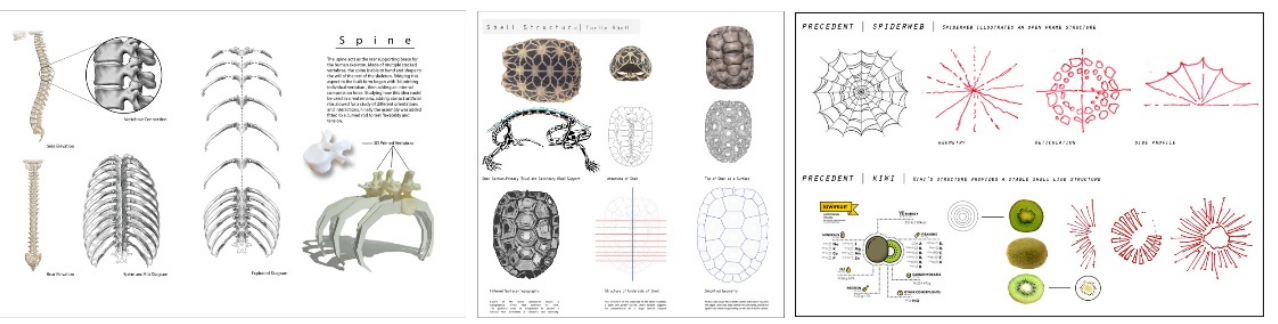

Fig. 1. Geometry, pattern, and structure in nature. Analytical drawing. Student Work from author's taught studio.

\subsection{Project: 2D Image to Solid-Void Tactile Pattern}

The highlight of this project was to observe texture from nature and man-made and manipulate in Photoshop to recreate that image in two or three shades that can be outlined and laser cut for tactile 3D model. The figure below shows original texture from grass blade and carpet transformed to two-dimensional tactile patterns. The primary objective was to observe, extract, manipulate, and recompose solid-void pattern. Techniques included Photoshop filter manipulation, outlining in Illustrator, and conversion to 2D AutoCAD for laser cut

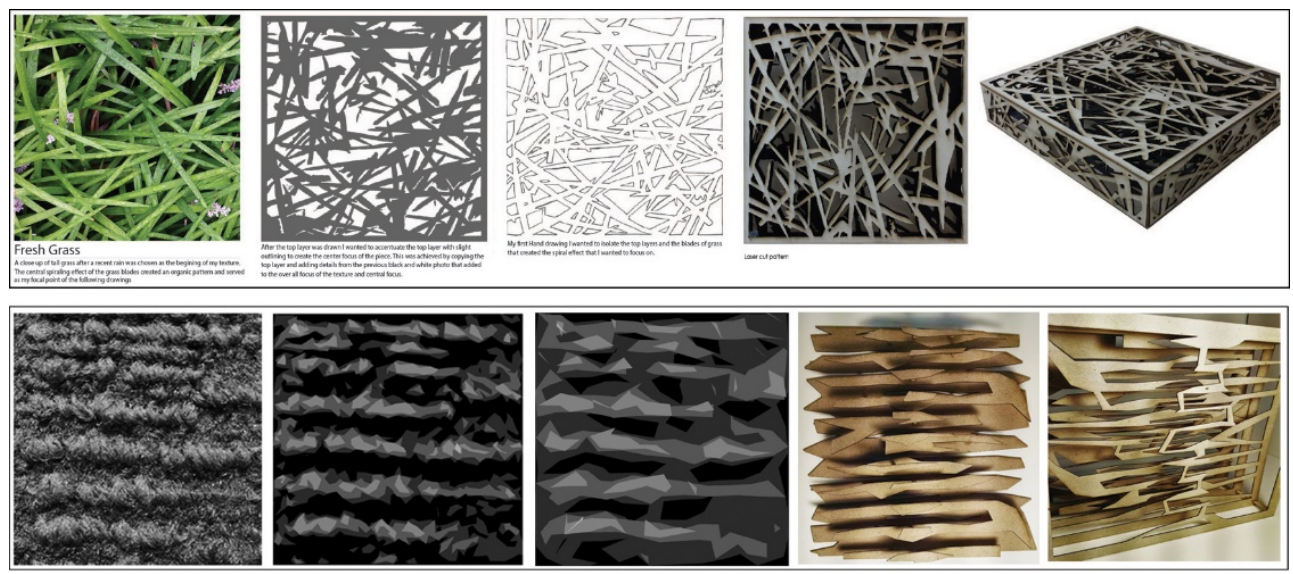

Fig. 2. 2D image from nature (grass blades) and man-made (carpet) manipulated and re-proportioned in to solid-void tactile fabrication pattern (author's $2^{\text {nd }}$ year studio student work). 


\subsection{Project: Module and Pattern}

The goal for this project was to understand the geometry of an individual element and its relationship with other elements to make a module that can be repeated as a tile. The start of this project was to manually draw individual components and to compose them in module for a desired pattern in solid-void. Techniques included hand drawing, Photoshop manipulation, outlining in Illustrator, and conversion to AutoCAD for laser cut.

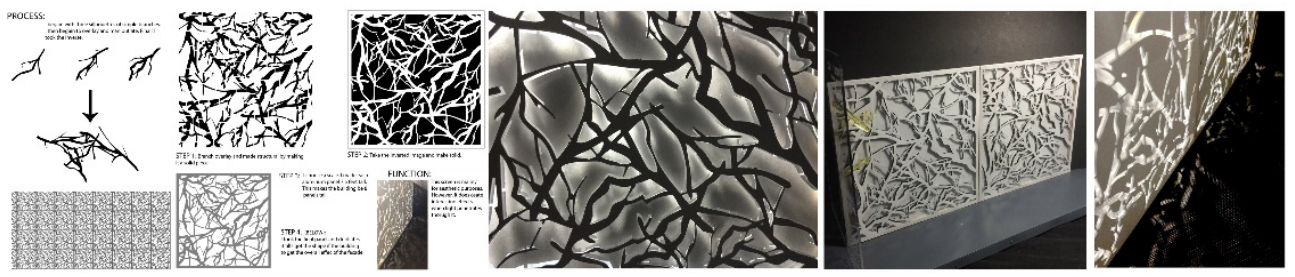

Fig. 3. The geometry of three tree branches overlapped and composed in hand drawing and then converted to CAD drawing for laser cut (Author's $2^{\text {nd }}$ year studio student work)

\subsection{Project: 2D Platonic Geometry to 3D KineticPattern}

Below are two projects. They demonstrate exploration of platonic geometry (hexagon and rectangle) to form expandable and retractable kinetic systems by reforming original geometries. Using additional elements of pivot points the modules were able to expand and contract in all directions. The working principle of this kinetic movement was entirely conceived by tectonic experimentation in physical pieces rather than digital models. Only AutoCAD and laser cutter were used to create these expandable modular systems. Developing a connection system became an important factor for these explorations.
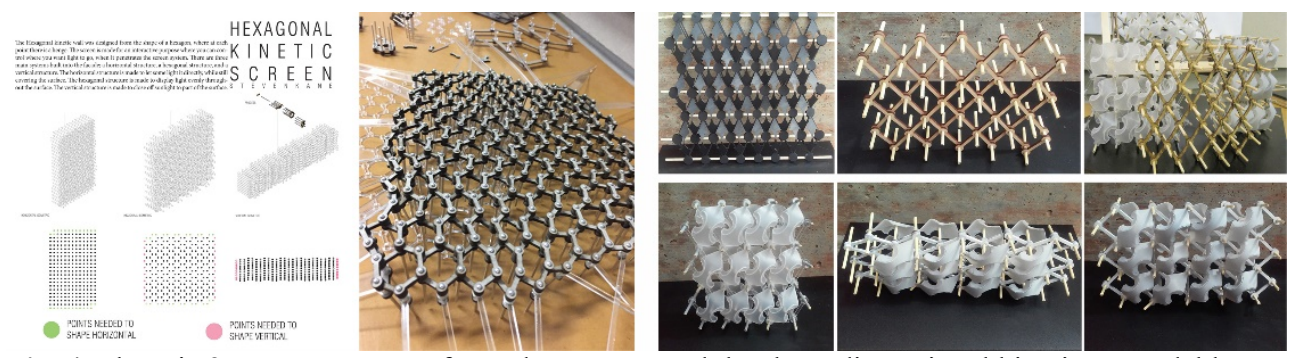

Fig. 4. Platonic 2D geometry transformed to create modular three-dimensional kinetic expandable systems through tactile exploration (author's $2^{\text {nd }}$ year studio student work) 


\subsection{Project: 3D Geometry from Human Bone Tissue}

This project takes lesson from microscopic human bone tissue formation and geometry formed by them. This exercise required detailed investigation, observation, and understanding in three dimensions. Since none of the surfaces are in a single plane the study had to be in Rhino 3D model and 3D print.
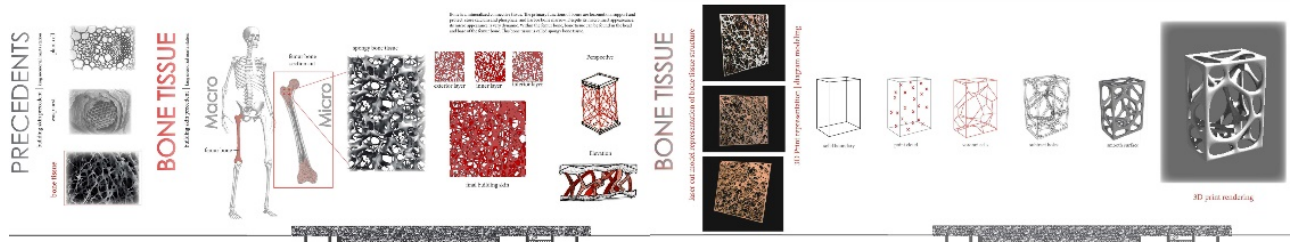

Fig. 5. Pattern extracted from bone tissue by $3 \mathrm{D}$ visualization and $3 \mathrm{D}$ print (author's $2^{\text {nd }}$ year studio student work)

\subsection{Project: Manual Mold Cast and Modular Wall System}

Connecting the same unit in various ways to create a modular patterned wall was the objective of this short one-week project. Students first had to design a single unit in a handcrafted model to create a female mold for casting. That mold was then used to cast several other copies of that same unit to construct the modular wall. The total process was executed entirely in manual techniques of mold casting. This is an effective substitute of 3D digital print.
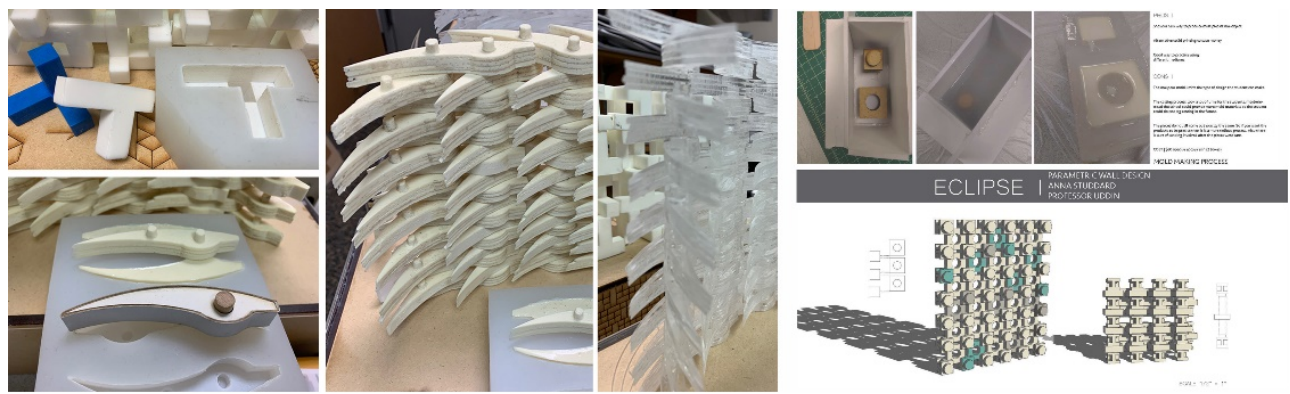

Fig. 6. Mold casting technique to fabricate modular wall system from a single unit (author's $2^{\text {nd }}$ year studio student work).

\subsection{Project: Kinetics from Nature}

Using inspiration from a Peacock's expandable feather system, this project analyzes the geometric pattern and constructs a 3D structural system that can expand and contract and span a large space. The fanned tail of a peacock presents geometry when diagrammed creates the mapping of a structural truss system. This system can be applied two-dimensionally as an expandable fan and three-dimensionally as an expandable dome. The method can be applied as a kinetic roofing or wall system that divides spaces according to program. It can also be applied as an expandable shell structure that can create temporary space. Analysis of geometry, kinetics, hand diagram and physical model iterations were the primary means for this exploration. 


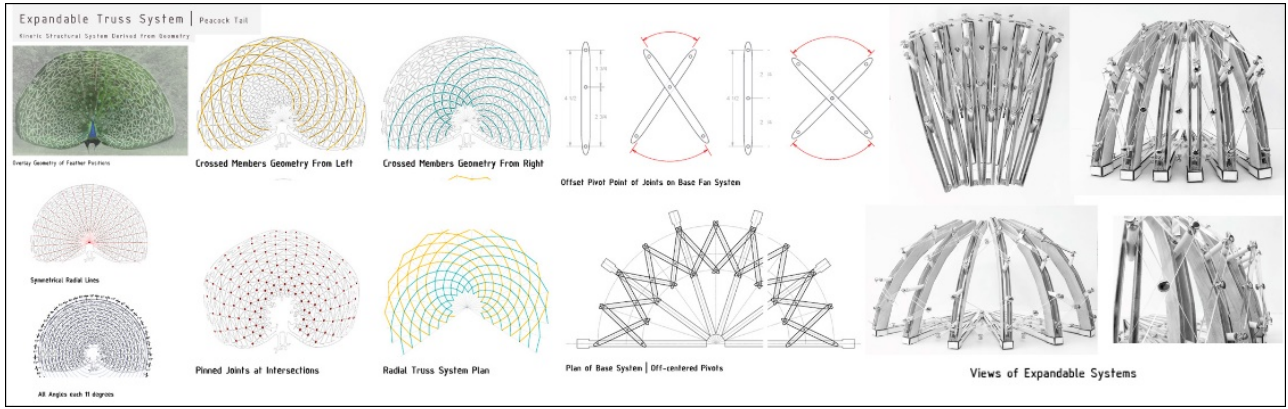

Fig. 7. Nature inspired pattern and kinetics for large span structure. Adaptation of transformable geometry in peacock's feather to design a structure with kinetic expandable truss system (author's $2^{\text {nd }}$ year studio student work).

\section{Conclusion}

The lack of understanding between virtual and actual (digital and analog), in particular understanding of 'scale' seems to be alarming in all levels of architectural studios. In the author's studio half of the students had difficulties in constructing a laser cut model in $1 / 8^{\text {th }}$ scale from a provided 3D digital file. Improper use of a 3D printer to cut simple pieces is becoming more common than before. These hints that the increasing use of digital tools has resulted in to decreasing the role of observation, analysis, and three-dimensional thinking in the beginning studios.
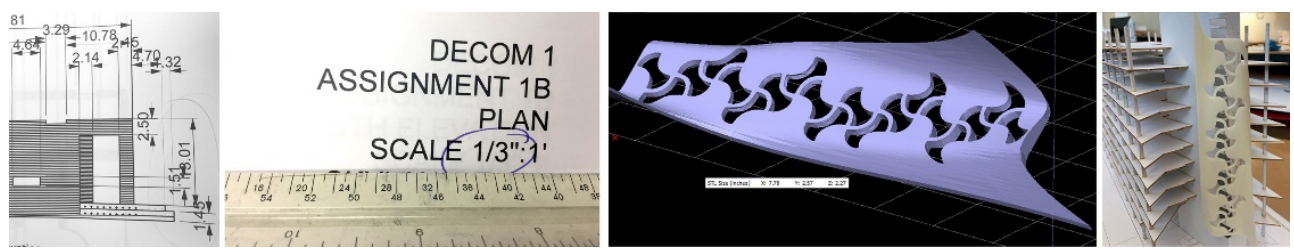

Fig. 8. Scale in print is vague and not within convention. Improper use of $3 \mathrm{D}$ printer demonstrates reluctance in exploration of other materials and techniques.

As mentioned before, architecture has the strongest educational ties to geometric organization because of the necessity for order and efficiency in construction. In that regard, understanding geometry and its order is of prime importance and thus should be studied before generating them in digital applications. Unfortunately, most architecture curriculums had taken out descriptive geometry courses in order to accommodate other courses primarily related to software learning.
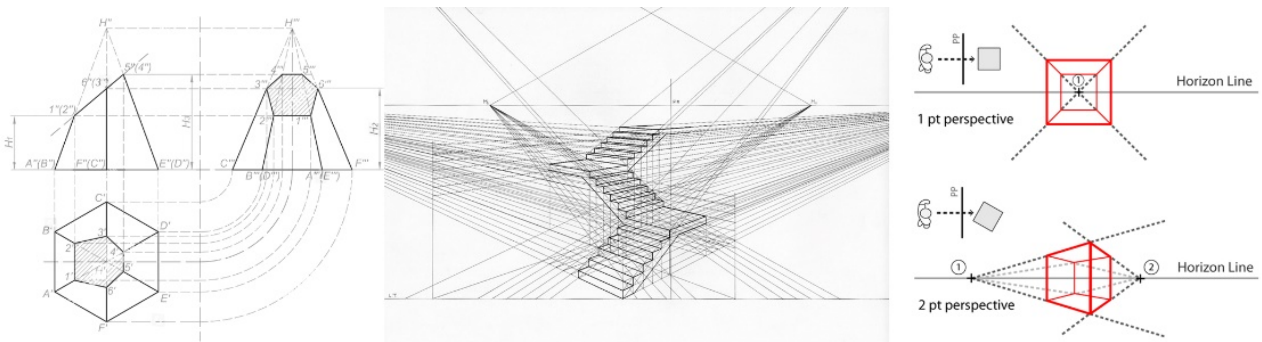

Fig. 9. Although descriptive geometry is no longer a part of architecture curriculum in most schools, it is the foundation of understanding geometry and three-dimensional thinking. 
The current trends of design education are becoming dependent on the ability of digital application rather than incorporation for principles of design, understanding of geometry, and aspects of other design forces. This includes program, site, environment, etc. The projects in this paper are short exercises running between two to three weeks demonstrating an effort to incorporate three-dimensional tactile design thinking through geometry and pattern. Digital applications are not ignored but used strategically when needed. 